\title{
Risiken von Supervision: Perspektiven in ein Dunkelfeld
}

\author{
Brigitte Schigl
}

Online publiziert: 20. Juli 2016

(C) Der/die Autor(en) 2016. Dieser Artikel ist eine Open-Access-Publikation.

Zusammenfassung Der Beitrag beschäftigt sich mit den Risiken von Supervision. Aus quantitativen wie qualitativen Daten von Supervisand_innen wie Supervisor_innen werden erste Hinweise auf das Dunkelfeld Supervision analysiert: Dabei können Prozessund Strukturmerkmale als Risikoquellen betrachtet werden, allen voran die Person der Supervisor_in, aber auch Gruppen- bzw. Teamkonstellationen mit ihren Dynamiken. Die Auftragsklärung und das Beachten der Organisationsdynamiken mit der Balance zwischen Schweigepflicht und Rückmeldung an die Auftraggeber_innen stellt dabei eine besondere Herausforderung dar. Schlussfolgerungen für Supervisor_innen, Berufsverbände und Supervisionsforschung werden vorgestellt.

Schlüsselwörter Supervision · Risiko · Schaden · Supervisionsforschung

\section{Risks of Supervision: First Views in the Darkfield}

Summary This article deals with the risks of clinical supervision. From quantitative and qualitative data given by supervisors and supervisees the author extracts first views in the darkfield of supervision: Process and structure are sources of risks - first the person of supervisor, then in group- and team constellations with their special dynamics. To be careful in the contracting process, consider the organisational dynamics, hold balance between confidentiality and

Die Arbeit ist Teil des Leitthemas „Supervision, Coaching und Psychotherapie“.

\section{B. Schigl ( $\bowtie)$}

Department für Psychotherapie und biopsychosoziale

Gesundheit, Donau Universität Krems,

Rembrandtstraße 4/11, 1020 Wien, Österreich

brigitte.schigl@aon.at feedback for the contracting organisation are special tasks for all supervisors. Conclusions for practitioners, for the professional associations of supervisors and supervision research will be drawn at the end of the article.

Keywords Supervision · Risk · Damage - Supervision Research

\section{Einführung: Supervisionsforschung im Dunkel- feld}

Supervision tritt an um die Qualität von Arbeitsprozessen, v. a. in Bezug auf die Interaktion zwischen Kolleg_innen, Mitarbeiter_innen, Klient_innen oder Patient_innen etc. zu erhöhen. Dazu wird seit den 1980er Jahren Supervisionsforschung betrieben, die v. a. die Benefits von Supervision im Blick hat (z. B. DGSv 2008). Das früher oft beklagte Forschungsdefizit für Supervision ist einer Vielzahl von Studien gewichen - meist Abschluss- bzw. Masterarbeiten von Studierenden in Supervisionsausbildungen. Diese kleinteiligen Untersuchungen spiegeln die Heterogenität des Feldes und des Formats wieder: Team-, Gruppen- oder Einzelsupervision, Organisationssupervision und Coaching für Führungskräfte, Supervision als Teil der Ausbildung, als verpflichtender Arbeitsauftrag oder aus eigenen Antrieb in Anspruch genommen, als regelmäßige Dienstleistung, sporadisch oder nur in Krisenfällen zugekauft, von Arbeitgeber_innen oder den Supervisand_innen finanziert, im klinischen, psychosozialen, pädagogischen, kulturellen, wissenschaftlichen, verwaltenden und manchmal auch Profit Bereich, von Supervisor_innen unterschiedlicher Herkunftsberufe und Erfahrungen mit unterschiedlichen Methoden und Zielsetzungen durchgeführt: „Die eine“ Supervision existiert nicht (Petzold et al. 2003), ebensowenig wie einheitliche 
Forschungsdesigns und geordnete Themenfelder. Oft fehlt auch die Einbettung in einen wissenschaftlichen Kontext. Die Komplexität von Supervision mit ihren multidirektionalen Einflussfaktoren und spezifischen Feld- und Auftragsbedingungen widersetzt sich einer Vereinheitlichung (Schigl 2004; Hausinger 2009). Die meisten der vorgestellten Arbeiten zum Nutzen des Formats stellen Supervision ein gutes Zeugnis aus und berichten über die Zufriedenheit der Supervisand_innen bzw. machen Qualitätskriterien geglückter Supervision fest (z. B. Schigl 2007; Möller 2012).

Meist werden diese Ergebnisse durch eine post hoc Befragung der Supervisand_innen oder Supervisor_innen erzielt. Oft sind es dabei die Supervisor_innen selbst, die die Befragung initiieren. Wenige Studien werden von neutralen Dritten durchgeführt, kontrollierte Studien blieben bisher Desiderat. Problematische Verläufe von Supervision, unerwünschte Effekte, Nebenwirkungen und Schäden von Supervision werden kaum thematisiert (Leitner et al. 2004). Diese Themen in Psychotherapie und Beratung zu beleuchten ist ein Forschungs-Schwerpunkt am Department für Psychotherapie und biopsychosoziale Gesundheit an der Donau Universität Krems. Neben der großen RISK-Studie für die Psychotherapie (Leitner et al. 2014) wurden mithilfe Studierender im Lehrgang Supervision \& Coaching auch Daten zum Dunkelfeld in der Supervision erhoben.

Hinweise auf eine doch mangelnde Qualität von Supervision geben länderübergreifende Untersuchungen im Feld der Geriatrie: Dort schrieben in einer breiten Umfrage $(n=354)$ nur ca. ein Drittel der Supervisand_innen ihrer Supervision einen hohen persönlichen Nutzen zu. Die gerontologische Feldund Fachkompetenz der Supervisor_innen wurde von nur einem knappen Drittel (!) der Befragten als hoch beurteilt (Petzold et al. 2008). Ähnliche Ergebnisse liefern Felderkundungen in der Psychiatrie (Gottfried 2012): $20 \%$ der Befragten geben in einer multinationalen empirischen Erhebung Negativerfahrungen mit Supervision an. Buchinger (2015) fand in einer breiten Umfrage ( $n=993$ ) im Profit Bereich, dass von 456 Personen, die Beratung in Anspruch nahmen, ca. $12 \%$ keinen Nutzen daraus ziehen konnten und ca. $5 \%$ fanden, dass sich die Situation durch die Intervention noch verschlimmert hat. Dies deckt sich mit den Untersuchungen im Bereich der Psychotherapie, die (je nach Autor_in) 10-20\% problematische Verläufe von psychotherapeutischen Behandlungen hochrechnen (z. B. Kraus et al. 2011).

Die im Folgenden behandelte Supervisionsthematik bezieht sich v. a. auf Supervisionen im psychosozialen und sozialpädagogischen Bereich.

\section{Das Dunkelfeld: Was sind unerwünschte Effekte?}

Als Nebenwirkungen in der Supervision können wir unerwünschte und unbeabsichtigte Effekte betrach- ten, die durch die Supervision auftreten. Dieses Risiko von (unerwünschten) Nebenwirkungen besteht bei jeder Intervention mit Menschen (Schigl 2011) - kein/e Supervisor_in kann immer im Blick haben, welche Folgen seine/ihre verbalen und nonverbalen Interventionen bei jeder $/ \mathrm{m}$ einzelne/n Supervisand_in haben können. Manchmal sind Verschlimmerungen auch Dynamiken geschuldet, die außerhalb des Bereichs der Supervision liegen. Dann allerdings sollte der/die Supervisor_in das erkennen und rückmelden. Wenn aber mangelnde Kompetenz, mangelnde ethische Haltung oder die Persönlichkeitsstruktur des/der Supervisor_in eine Metakommunikation über Unzufriedenheiten und Irritationen unmöglich machen (Ehrhardt und Petzold 2011) und eine Verständigung nicht gelingt, so kann die (Neben)wirkung als Fehler des/der Supervisor_in betrachtet werden. Auch ohne gemeinsame meta-kommunikative Bearbeitung können solch unerwünschte Wirkungen manchmal noch vom supervidierten System aufgefangen werden. Gelingt dies aber nicht, so können sie Schaden anrichten. Dieser Schaden kann sich schlimmstenfalls in psychischen Verletzungen und der Beeinträchtigung der Integrität einzelner oder mehrerer Supervisand_innen äußern (Ehrhard 2010). Er kann auch auf einer materiellen Ebene liegen, wenn Supervision Ressourcen (Arbeitszeit, Geld) verschlingt, ohne die Situation zu verbessern. Schaden entsteht auch durch eine Störung des supervidierten (Teil)Systems, wie z. B. einer Beeinträchtigung von Abläufen, Führungsoder Teamaufgaben (Keiblinger 2012).

\section{Ebenen von Risiken in der Supervision}

In der Beschreibung bzw. Beurteilung von Supervision kann man zwischen Struktur-, Prozess- und Ergebnisqualität unterscheiden. Zur Strukturebene zählen alle konstituierenden Elemente wie Person und Kompetenzen des/der Supervisor_in, die Auftragsdefinition Setting, Methoden etc.. Mit Prozessebene sind alle Phänomene der fortlaufenden Interaktionen gemeint, also wie die Sitzungen konkret ablaufen, wie sich die Beziehung zwischen Supervisor_in und Supervisand_in(nen) gestaltet, wie die Interventionen gesetzt werden, wie kommuniziert wird etc.. Die Ergebnisqualität befindet darüber, ob bzw. in welchem Ausmaß das Ziel der Supervision erreicht wurde, wie die Zufriedenheit aller Beteiligten mit der Supervision ist. Petzold erhebt eine noch weitergehende Forderung für die Ergebnisqualität: Ist Supervision auch in der Arbeit mit Kund_innen/Klient_innen/Patient_innen, also auf der Ebene der End,,konsument_innen“ von Dienstleistungen und Behandlungen sichtbar (Petzold et al. 2003 S 231)?

$\mathrm{Zu}$ allen drei Bereichen von Supervision konnten Hinweise für Risiken und mangelhafte Qualität erhoben werden. Die nun präsentierten Überlegungen basieren auf einer Synopsis der Autorin aus den qualitativen und quantitativen empirischen Daten 
von Judith Kero (2010 - Literaturanalyse zu Risiken von Supervision und Coaching in den Zeitschriften „OSC“ und „supervision“), Sabine Karlinger (2010 Genderkompetenz von SupervisorInnen aus Sicht der Supervisand_innen), Heidemarie Hinterwallner (2010 - (Un)erwünschte Wirkungen aus Sicht von Sozialarbeiter_innen), Margareta Keiblinger (2012 Risiken, Schäden aus Sicht von Lehrsupervisor_innen) und der Online-Dunkelfeldstudie „Verletzungen in der Supervision und Lehrsupervision" von Jutta Ehrhardt und Hilarion Petzold mit Daten von Supervisand_innen (Ehrhardt und Petzold 2011). Die Mehrzahl der Forschungsprojekte entstanden als Abschlussarbeiten im Lehrgang Supervision \& Coaching der Donau Universität Krems und stellen die größte Datenmenge und -vielfalt zu diesem Thema in der deutschsprachigen Supervisionsforschung zur Verfügung.

Aus den oben genannten Datenquellen lassen sich in einer Triangulation folgende Risiko-Bereiche erheben (Reihung nach Häufigkeit der Nennungen in den Daten):

\section{Strukturvariable:}

- Persönlichkeit des/der Supervisor_in

- Diagnoseerstellung und Auftragsklärung, Settingwahl

- Feldkompetenz des/der Supervisor_in

- Fachkompetenz des/der Supervisor_in, Genderund Diversitykompetenz

2. Prozessvariable:

- Passung

- Metareflexion und Metakommunikation

- Berücksichtigung der Organisationsperspektive

- Umgang mit der Gruppen- bzw. Teamdynamik

- Interventionen, Einsatz von Methoden und Medien

- Konfrontationsbereitschaft des/der Supervisor_in

- Verschwiegenheit

3. Ergebnisqualität/Zielerreichung

Nun zu den einzelnen Punkten:

\section{Risiken/Mängel auf der Strukturebene liegen in ...}

\section{Persönlichkeit des/der Supervisor_in}

Das höchste Risiko bringen die Expert_innen (Keiblinger 2012) mit der Person und der Persönlichkeit der Supervisor_innen in Verbindung. Sie nennen deren Machtstreben, Geltungsdrang, Selbstüberschätzung Überheblichkeit oder auch Bedürftigkeit nach Anerkennung als Risikopotentiale. Meistens werden diese Phänomene narzisstischen Persönlichkeitsstrukturen der Supervisor_innen zugeschrieben und eine damit verbundene mangelnde Wertschätzung der Supervisand_innen konstatiert.

\section{Diagnoseerstellung und Auftragsklärung}

Aus Interviews (Hinterwallner 2010; Keiblinger 2012) geht hervor, dass ein unbedacht übernommener Auftrag einen hohen Risikofaktor darstellt. Eine sorgfältige Aushandlung des Auftrags, eine gemeinsame Diagnoseerstellung mit den Auftraggeber_innen findet dabei nicht statt. Immer wieder kommt es vor, dass Supervision nicht das passende Format für das präsentierte Problem ist: Das „Trostpflaster“ Supervision ersetzt eben nicht mangelnde Qualifikation oder Überforderung von Mitarbeiter_innen, Personalund Ressourcenknappheit, Mediation in eskalierten Konflikten, Verbesserung der Führungsqualität etwa durch Leitungscoaching oder eine Organisationsentwicklung. Auch kontinuierliches Klagen über die schwierigen Arbeitsbedingungen in der Supervision als „Psychohygiene“ interpretiert, wäre ein falsch verstandener Auftrag. Aus unbedacht übernommen Aufträgen können Fehl-Festlegungen des Settings bzw. der Ziele resultieren. V. a. Supervisions-Berufsanfänger_innen sind gefährdet, mangelhaft geklärte Supervisionsaufträge $\mathrm{zu}$ übernehmen (Keiblinger 2012). Supervisand_innen beklagen die oft mangelnde Fokussierung in der Supervision (Hinterwallner 2010).

\section{Settingwahl}

Die Frage, wer an der Supervision teilnimmt bzw. wie lange diese dauert, ist eng mit der Auftragsklärung verbunden. Zentral für Teamsupervisionen ist die dabei laut Expert_innenaussagen die Frage, ob bzw. inwieweit die Leitung eingebunden wird. Viele Expert_innen sind sich einig, dass ohne diese Einbindung der Führungsebene Supervision keine Änderung von Missständen bringen könne (Keiblinger 2012). Probleme, die sich in den Supervisionen manifestieren, liegen eben oft auf anderen Hierarchieebenen begründet und können auf der Ebene, auf der die Supervision stattfindet höchstens teilweise befriedigend bearbeitet werden. Bei alleiniger Arbeit mit dem Team kann Supervision auch dazu führen, dass sich Fronten zwischen Team und Leitung verhärten.

Eine andere Perspektive bringen dazu die von Ehrhardt (2010) befragten Supervisand_innen: Teamsupervisionen im Beisein der Leitung bergen ein besonders hohes Verletzungsrisiko. Eine Einbindung der Leitung in Form von Gesprächen und Informationsweitergabe durch die Supervisor_in kann laut Supervidand_innen auch eine Verletzung der Schweigepflicht bedeuten.

\section{Fach- und Feldkompetenz, Gender- und Diversitykompetenz}

Als Fachkompetenz kann man das Wissen um den Theorie- und Forschungsstand von Supervision generell bezeichnen. Wichtig laut Expert_innenmeinung ist hier eine qualitativ hochwertige Supervisionsausbildung, die die Besonderheiten des Formats 
vermittelt und nicht in personalisierten Interventionen hängen bleiben darf (Keiblinger 2012).

Feldkompetenz kennzeichnet das Wissen um die spezifischen Bedingungen und Themen eines bestimmten Berufsfeldes. Ist sie zuwenig vorhanden steigt das Risiko, dass die Dynamik eines spezifischen Arbeitsfeldes nicht erkannt wird. Mehrfach wird hier das klinische oder sozialpädagogische Feld genannt. Eine zu hohe Felderfahrung wird aber auch als Risiko gesehen, wenn die Supervisor_innen mehr als Fachberater_innen fungieren und ihre Supervisand_innen „lehren“ wollen, wie sie zu tun hätten (Keiblinger 2012). Eine solche „Betriebsblindheit“ durch zu große Nähe zum Feld wurde auch von den interviewten Sozialarbeiter_innen als Risikofaktor für die Supervision betrachtet (Hinterwallner 2010).

Gender- und Diversity-Kompetenz müssen, so Abdul-Hussain (2012) mit supervisorischer Fachkompetenz verknüpft werden (S 56). Eine solche Gender- und Diversitykompetenz wird von Supervisand_innen jedoch erst wahrgenommen, wenn diese selbst für diese Themen sensibilisiert sind (Karlinger 2010), ansonsten äußert sich ihr Mangel nur in einem diffusen Unbehagen.

\section{Risiken/Mängel auf der Prozessebene liegen in ...}

\section{Passung}

In den Expert_innenaussagen ist mit „Passung“ die Qualität der supervisorischen (Arbeits)beziehung gemeint. Diese entsteht durch das Zusammenwirken der Persönlichkeit, der fachlichen und sozialen Kompetenzen und der Haltung des/der Supervisor_in in Verbindung mit jenen der Supervisand_innen. Besonders im intimeren Einzelsetting ist diese Passung ausschlaggebend; in der Teamsupervision sind etwa feldunübliche Sprache, Kleidung oder ein irritierendes Auftreten der Supervisor_innen Faktoren, die Arbeitsbeziehung schwierig(er) machen (Keiblinger 2012).

\section{Metareflexion und -kommunikation}

Die Expert_innen sind der Meinung, dass supervisorische Tätigkeit eine eigene Supervision der Supervisor_innen implizieren muss (Keiblinger 2012). Auch bei langjähriger Praxiserfahrung ist es nötig, sein eigenes Handeln immer wieder zu reflektieren, die eigenen blinden Flecken zu suchen und zu korrigieren.

Den wichtigsten Faktor für eine Minimierung von Fehlerfolgen stellt die Metakommunikation mit den Supervisand_innen dar - entweder als Resultat eigenen Reflektierens oder oben genannter Supervisionsoder Intervisionserkenntnisse; oder auch als ad hoc Reaktion auf von Supervisand_innen angesprochene Irritationen oder Problematiken in der Beziehung. Auch hier ist eine Parallele zu den Erkenntnissen der RISK Studie für Psychotherapie gegeben (Schigl 2014a).

\section{Individuumzentriert statt Organisationsbezug}

Dieser Aspekt wurde von den Expert_innen mehrfach betont: Supervision darf nicht allein auf die Perspektive Individuum oder Team beschränkt bleiben, sondern muss Organisation und Feld, oft auch gesellschaftliche Makroebenen miteinbeziehen. Die mangelnde Beachtung der Struktur und Dynamik der Gesamtorganisation wird als wichtige Fehlerquelle bei Supervisionen gesehen. Schlechte Erfahrungen wurden dabei von Psychotherapeut_innen, die Supervision ohne supervisorische Zusatzausbildung anbieten, rückgemeldet (Keiblinger 2012). Auch in den Daten Ehrhardts (2010) tritt zutage, dass überall dort, wo die Person des/der Supervisand_in (zu sehr) im Mittelpunkt stand, die größte Zahl der Verletzungen zu verzeichnen ist. Die von Hinterwallner (2010) befragten Sozialarbeiter_innen nannten dazu Bevorzugungen, Regelverletzungen, Bewertungen.

\section{Umgang mit Gruppen- bzw. Teamdynamik}

Diese Risikoperspektive bezieht sich auf das supervidierte Teilsystem einer Organisation bzw. ist maßgeblich in der Gruppensupervision, besonders der Supervision in Ausbildungskontexten (Lehrsupervision, Praxissupervision). Die Konkurrenz der Teilnehmer_innen kann dort eskalieren, wenn Supervisor_innen nicht achtsam sind. Die Expert_innen (Keiblinger 2012) berichteten vom Ignorieren gruppendynamischer Prozesse oder Entgleisungen von Feedbackrunden, wenn für einen/eine Protagonist_in eine kritische Rückmeldung nach der anderen kommt, ohne dass der/die Supervisor_in eingreift (oft sogar mitmacht!). Hier ist die die Gefahr von persönlichen Kränkungen und Bloßstellung für die Supervisand_innen sehr groß. Bei solchen Grenzverletzungen verlangen Supervisand_innen von ihren Supervisor_innen Schutz und Vorbild zu sein (Hinterwallner 2010).

Auch der Umgang mit der Team-Dynamik in Bezug auf die Leitungsebene fällt unter diesen Risikofaktor - etwa wenn Supervisor_innen sich mit dem supervidierten Team gegen deren Führungsebene verbünden, oder sich als die „besseren Leiter_innen“ fühlen, statt beide Perspektiven und die Gesamtorganisation im Auge zu haben (Keiblinger 2012).

Gruppendynamische Prozesse sind also Risiko wenn sie den Blick auf die/den Einzelne/n verstellen oder umgekehrt die Dynamik der Gesamtorganisation in den Hintergrund treten lassen.

\section{Parteilichkeiten und Beziehungsdynamiken}

Wenn Supervisor_innen ihre Allparteilichkeit verlassen stellt das ein Risikopotential dar. Sei es durch eine schon bestehende (enge) Beziehung zu Führungspersonen (die vielleicht den/die Supervisor_in beauftragt haben) oder in Bevorzugen oder Ablehnen einzelner Supervisand_innen (Ehrhardt 2010). Es ist hier besonders die Teamsupervision am Arbeitsplatz, die hohes Verletzungspotential birgt. 
Jungmeiers (2011) Daten von Supervisand_innen lassen auf tendenziell ein höheres Maß an Unzufriedenheit bei internen, also organisationsangehörigen Supervisor_innen schließen. Dies war besonders deutlich im Setting der Einzel- und Gruppensupervisionen. Hier könnte die Involvierung der internen Supervisor_innen in die Organisation Grund für die geringere Zufriedenheit im Vergleich zu den externen Supervisionsanbieter_innen sein.

\section{Interventionen und Methoden}

Ein für das Supervisions-Ziel und die Situation inadäquater Einsatz von Interventionen und Methoden stellt ein weiteres Risiko dar. Dieser Risikofaktor hat einen hohen Bezug zu dem Strukturfaktor Fachkompetenz der Supervisor_innen. Falsch ist es laut Expert_innen z. B. supervisorische Schwierigkeiten immer als „Widerstand“ der Supervisand_innen zu interpretieren (Keiblinger 2012). Die ausschließlich tiefenpsychologische Deutung von Beziehungsdynamiken als Übertragung und Gegenübertragung ist ein weiteres Beispiel für zu kurz greifende Intervention (Kero 2010).

Hinterwallners (2010) Ergebnisse betonen, dass Supervisand_innen die Sinnhaftigkeit der eingesetzten Methoden einsichtig sein muss. Die von ihr interviewten Supervisnd_innen beklagen mangelndes Zeitmanagement, falsche Settingwahl, zu starke Themen-Fokussierung, Eintönigkeit und Methodenarmut sowie unkontrollierte Feedbackrunden als Interventionsfehler.

\section{Schweigepflicht/Diskretion}

Expert_innen wie Supervisand_innen thematisieren diesen Punkt. Schweigepflicht bezieht sich sowohl auf die Geschehnisse mit den Supervisonsteilnehmer_innen, als auch darauf, was dort über über Kund_innen, Klient_innen oder Patient_innen gesprochen wurde. Schon das „Nachbesprechen“ von in der Supervision bearbeiteten Themen zwischen den Sitzungen kann sich als risikoreich erweisen. In den Daten der Supervisand_innen (Ehrhardt 2010) finden sich v. a. Beispiele für (vermutete, gefürchtete?) Schweigepflichtsverletzungen durch Supervisor_innen. Dieses Risiko ist besonders groß, wenn ein/e Supervisor_in in mehreren Organisations-Bereichen oder auf verschiedenen Hierarchieebenen tätig ist.

\section{Mangelnde Konfrontationsbereitschaft}

Hier haben wir einige Hinweise aus den Daten von Karlinger (2010), dass das Nicht-Ansprechen von offensichtlichen Konflikten oder schwierigen Dynamiken von Superviand_innen als Fehler gesehen wird. Auch Ehrhardts (2010) Daten lassen darauf schließen, dass Supervisor_innen sich nicht immer gruppendynamischen Prozessen entgegenstellen, sondern oft auch (auf Kosten einzelner Supervisand_innen) mit dem Strom schwimmen und Verletzungen zulassen.

\section{Mangelnde Ergebnisqualität}

Expert_innen (Keiblinger 2012) sehen in zu langer oder unhinterfragt andauernder berufsbegleitender Supervision ein Fehlerrisiko. Wenn nicht von Zeit zu Zeit evaluiert wird und hinterfragt, was denn der Auftrag der Supervision weiter ist, können Prozesse versanden oder der Auftrag aus dem Auge verloren werden. Die Ergebnisqualität der in vielen psychosozialen Einrichtungen gängigen permanenten berufsbegleitenden Supervision ohne klare Ziele wird hier sehr kritisch gesehen.

\section{Verletzungen durch Supervision}

In einem quantitativen Datensatz von 154 Supervisand_innen v. a. aus dem psychosozialen Bereich (60\% weiblich, $28 \%$ männlich $12 \%$ o. A.), die mittels Onlinefragebogen zu Verletzungen in Supervision und Lehrsupervision befragt wurden, zeichnet sich ein aufrüttelndes Ergebnis ab (Ehrhardt 2010; Ehrhardt und Petzold 2011): Als besonders „gefährlich“ konnte hier das Mehrpersonen-Setting extrahiert werden, in dem Kränkungen, Demütigungen, Entwertungen und Bloßstellungen als Verletzungen berichtet werden. Im Einzelsetting dagegen sind Grenzüberschreitungen und Kränkungen am häufigsten genannt. Besonders risikoreich für Verletzungen erweist sich verpflichtende Supervision, die am Arbeitsplatz stattfindet; dies waren in der Befragung v. a. Einrichtungen aus dem stationären Bereich. Teamsupervision birgt die am schwersten empfundenen Verletzungen. Wenn Vorgesetzte an der Supervision teilnehmen, erhöht dies das Verletzungsrisiko. Dabei haben die so gekränkten Supervisand_innen auch keine Unterstützung im Team erhalten, sondern fühlten sich alleingelassen. Fast die Hälfte der Befragten gaben wiederholte Verletzungen an. Die Herkunftsberufe der verletzenden Supervisor_innen waren v. a. Psycholog_innen (38\%) und Sozialarbeiter_innen (26\%). Nach Häufigkeit ranggereiht sind es v. a. psychoanalytisch orientierte, systemische und gruppendynamisch orientierte Supervisor_innen, die am meisten verletzten. Männliche Supervisoren wurden deutlich häufiger verletzend erlebt als weibliche und kränken am häufigsten durch Demütigung, Bloßstellen und Grenzüberschreitungen; weibliche Supervisorinnen durch das Zuschreiben von Inkompetenz. Als vermutete Gründe für das verletzende Verhalten ihrer Supervisor_innen gibt die überwiegende Mehrheit deren Eitelkeit (84\%) an, $43 \%$ nennen die Verletzung einen „technischen Fehler" und $39 \%$ führen sie auf Rivalität und Konkurrenz im Mehrpersonen-Setting zurück. Über $75 \%$ der Befragten gaben an, dass eine Klärung mit den Supervisor_innen nicht möglich war. Bei fast der Hälfte der Daten (49\%) wurden die Verletzungen als „bedeutsam“ bis „schwer“ angegeben. Als häufigste Bewältigungsmethoden wurde ein Besprechen im eigenen Freundeskreis genannt, Supervisand_innen müssen 
also das Arbeitsfeld verlassen und sich außerhalb Entlastung suchen. Viel weniger Supervisand_innen nennen eine Entwertung des/der Supervisor_in als Reaktion auf die zugefügten Verletzungen. Bei der Verletzungsart Grenzüberschreitung wird am häufigsten nach professioneller externer Unterstützung nachgefragt. Bei $5 \%$ der Befragten war ein Verlassen der Arbeitsstelle (Versetzung, Kündigung) die Folge der Verletzungen. Weiters führte Ehrhardt im Rahmen ihrer Untersuchung qualitative Interviews mit zu Schaden gekommenen Supervisand_innen durch und vertiefte die quantitativen Ergebnisse.

Natürlich müssen diese Daten vorsichtig interpretiert werden - die Studie forderte ja auf, über Verletzungen in der Supervision zu berichten und hat solche Proband_innen angezogen, die derartiges erlebt haben. Die Stichprobe ist also nicht repräsentativ, sondern als Streiflicht in ein Dunkelfeld zu verstehen. Die Aussagen sind jedoch jenseits von statistischen Parametern wichtig und werden inhaltlich auch durch die Kategorien aus anderen Datenquellen bestätigt (vgl. Abschn. 3).

\section{Zusammenfassung und Schlussfolgerungen}

Vorausgeschickt muss werden - darin sind sich alle Expert_innen einig - dass auch bei einer nach dem State of the Art durchgeführten Supervision schwierige Situationen auftreten können (vgl. Pühl 2009).

Aus der Zusammenschau der Daten geht eindeutig hervor, dass Supervision Risiken birgt und Schäden hervorrufen kann. Supervisor_innen sind sich dieser ihrer Macht aber nur wenig bewusst (Petzold 2009). Alle im Supervisionsprozess maßgeblichen Faktoren auf Struktur- und Prozessebene können Risiken bergen: Fehler geschehen am häufigsten durch mangelnde Fach- und Feldkompetenz und -performanz also im Grunde Selbstüberschätzung - der Supervisor_innen. Ihre Persönlichkeit, hier v. a. narzisstische und Macht-Thematiken bzw. mangelnde Auseinandersetzungs- und Konfliktfähigkeit sind Quellen von Verletzungen. Auf der Prozessebene bergen die Dynamiken in den supervidierten Teams und Gruppen Gefahren: Die oft schwer durchschaubaren multimotivationalen Strömungen wie Hierarchie- und Rangbestimmungen, Atmosphären von emotionaler Ansteckung, Gruppennormen und Gruppendruck, Isolierung und Spaltungen, kommunikativen Bemächtigungsstrategien und selbstwerterhaltenden Argumentationen können für Supervisand_innen schädigend sein, wenn ihre Supervisor_innen dies nicht erkennen und eingreifen. Besonders brisant können solche Dynamiken in Lehr- und Ausbildungssupervisionen sein, in denen der Konkurrenz - und Profilierungsdruck hoch ist (vgl. Beitrag über Lehrsupervision in dieser Ausgabe). Generell sollte in der Supervision eine Ressourcenorientierung statt Defizitsuche, eine salutogenetische statt pathogenetische Sichtweise angestrebt werden.
In der Zusammenschau der Daten ist neben vielen Übereinstimmungen auch eine Differenz zwischen den Aussagen der Supervisand_innen und der Supervisionsexpert_innen auszumachen. Supervisand_innen beklagen Verletzungen durch Indiskretion v. a. in jenen Settings, in denen Supervisor_innen auf mehreren Systemebenen arbeiten und Kontakt mit Leitungsebene haben. Dementgegen fordern die Expert_innen idealiter Kontakt der Supervisor_innen auf mehreren Hierarchieebenen der supervisierten Organisation und eine Vernetzung mit den Führungspersonen der supervidierten Subsysteme. Gemeinsam ist den Aussagen aber die Betonung der Wichtigkeit der Transparenz: Was, mit wem und warum kommuniziert wird, muss offen gelegt werden, wenn Supervisor_innen in mehreren Organisations-Bereichen oder Hierarchieebenen tätig sind.

Über individuelle Verletzungen fehllaufender Prozesse hinaus sehen Supervisionsexpert_innen in ergebnislosen Supervisionen Schäden für die Auftraggeber_innen, zumeist Organisationen: Wenn belastende Themen auf keine bearbeitbare Ebene gebracht werden (können), entsteht Schaden zumindest im Sinne von Ressourcenverschwendung: Arbeitszeit- und Supervisorenhonorar wird hier ohne Benefit investiert. Wirtschaftliche Beweggründe bei den Supervisor_innen können Ursache dafür sein, eine Supervision fortzuführen, obwohl das Ziel erreicht wurde bzw. nicht (besser) erreicht werden kann. Dies verdeutlicht, dass Supervisor_innen von einem/einer Auftraggeber_in unabhängig sein müssen, um nicht aus Parteilichkeit oder finanzieller Not Aufträge anzunehmen, die sie nicht erfüllen können oder die unsinnig sind.

$\mathrm{Zu}$ Schäden von Supervision in Organisationen zählen aber auch Verhärtungen in Konflikten innerhalb von Teams oder zwischen Teams und deren Leitungsebene.

Ergebnislos oder schlecht verlaufende Supervisionen prägen die Teams und Einzelne und lassen sie künftigen supervisorischen Interventionen sehr skeptisch oder resignativ gegenüberstehen - der Ruf des Formats hat gelitten und das Interventionspotential von Supervision ist beschädigt.

\section{Was können Supervisor_innen tun?}

Hauptpfeiler einer Risikominimierung ist wohl die Person der Supervisor_in. Hier gilt es sich und das eigene Handeln freundlich-kritisch in den Blick $\mathrm{zu}$ nehmen und systematisch zu reflektieren. Blinde Flecken müssen in eigener Supervision oder Intervision aufgehellt werden. Dazu ist Selbstsicherheit nötig, die eigene Fehlhandlungen eingestehen kann, und eine Fehlerfreundlichkeit, die Wege zu deren Bearbeitung öffnet: Denn falsch gesetzte oder unterlassene Interventionen führen erst dann zu Schäden, wenn sie nicht reflektiert, kommuniziert und korrigiert werden (Schigl und Gahleitner 2013). 
Was können Berufsverbände und Ausbildungsträger_innen tun?

Zum Unterschied zur Psychotherapie ist Supervision ja kaum gesetzlich geregelt. Eine Verpflichtung zu Qualitätskriterien wie Verschwiegenheit und Vertraulichkeit, sowie Eigensupervision ist derzeit für Supervisor_innen der ÖVS (Österreichische Vereinigung für Supervision) vom Berufsverband gefordert, ein Qualitätsentwicklungsgespräch soll dies überprüfen (ÖVS Internet: http://www.oevs.or.at/die-oevs/ qualitat/). Die Supervisor_innen des ÖBVP müssen 30 Arbeitseinheiten supervisionsbezogene Fortbildung, speziell begleitende Supervision innerhalb von 3 Jahren nachweisen können (ÖBVP Internet https://www.psychotherapie.at/sites/default/files/ files/arbeitsbereiche/Supervision-Aufnahmekriterien2013.pdf ) - fraglich ist, ob diese Forderung auch üb erprüft wird. Dass eine professionelle Reflexion fi xer Qualitätsstandard von Supervision sein muss, da rüber herrscht Einigkeit in der Supervisorischen Co mmunity - die deutlichere Kommunikation dieser Fo rderung an die Mitglieder könnte vielleicht noch me hr Bewusstsein dafür schaffen.

Eine besondere Rolle kommt den Ausbildungsträger_innen von Supervisionslehrgängen zu. Da die Person des/der Supervisor_in einen zentralen Risikofaktor von Supervision darstellt, liegt es an ihnen, ihre Kandidat_innen sorgsam auszuwählen. Selbsterfahrung und Selbstreflexion, Persönlichkeitsbildung und Sensibilitätsentwicklung sollten wesentliche Ziele von Lehrveranstaltungen und Lehrsupervisionen sein. Denn individuelle Selbstwertproblematiken verführen dazu, (verdeckt)mächtige Positionen wie die eines/einer Supervisor_in zu missbrauchen, um narzisstische Aufwertung zu generieren. Dabei können die oben genannten Fehler auftreten und schädigende Handlungen für Individuen und Organisationen gesetzt werden. Bezüglich Fachkompetenz ist darauf zu achten, dass multitheoretische bzw. -perspektivische supervisorische Modelle vermittelt werden. Solche sind für die Durchführung von Supervisionen unumgänglich; denn falsch wäre es z.B. supervisorische Schwierigkeiten immer als „Widerstand“ der Supervisand_innen zu interpretieren oder andere einseitige Interpretationen zur Erklärung von Phänomenen zu verwenden. Auch eine Sensibilisierung in Bezug auf Gender und Diversity ist immer mehr nötige supervisorische Kompetenz (Schigl 2014b).

Die bisher gewonnenen Erkenntnisse über unerwünschte Effekte und Wirkungen von Supervision sollten auch in die Supervisionsausbildungen einfließen, um dieses Dunkelfeld ins Bewusstsein zu rücken und Sensibilität dafür zu entwickeln.

\section{Was kann die Supervisionsforschung beitragen?}

Primäre Forderung an Supervisionsforschung ist es, selbst ein höheres Qualitätsniveau zu erreichen. Die derzeit vorherrschende Herangehensweise lässt sich großteils als Legitimationsforschung, oft auf simplem Niveau bezeichnen. Hier sollten unabhängige Studien und Felderkundungen sich der Qualität von Supervision widmen und in ihren Fragestellungen eventuelle unerwünschte Effekte nicht ausklammern. Forscher_innen und publizierende supervisorische Praktiker_innen sind aufgefordert, sich diesen Themen zuzuwenden und eine kritische Selbstreflexion in bester supervisorischer Tradition $\mathrm{zu}$ entwickeln. Denn auch über die Fehler und Schwierigkeiten zu berichten, hilft das Format weiter zu entwickeln. Es gilt wie in der Psychotherapie: Solange die Beschäftigung mit Nebenwirkungen und Missständen der supervisorischen Tätigkeit nicht auch beforscht und diskutiert wird, befindet sich diese noch in einem vorwissenschaftlichem Stadium. Ein Vorantreiben qualitativ hochwertiger Ergebnisse für positiv wie negativ wirksame Effekte von Supervision ist vonnöten.

\section{Schlusswort}

Wichtig ist im Auge zu behalten: Supervisor_innen sind nicht gefeit gegen Fehler und unerwünschten Dynamiken, die aus ihren Interventionen erwachsen. Meist kann bei Missverständnissen oder Konflikten aber noch meta-kommuniziert werden und die unerwünschte (Neben)wirkung so verhindert bzw. gering gehalten werden. Derartige Meta-Kommunikationen über unerwünschte, irritierende oder störende Effekte können, wenn sie sorgsam aufgegriffen und bearbeitet werden, sogar zu einer Verbesserung des Prozesses führen oder Hinweise auf verborgene Themen geben (Schigl und Gahleitner 2013). Seien wir also bei uns wie bei unseren Supervisand_innen fehlerfreundlich und reflexionswillig.

Open access funding provided by Danube Universtity Krems University for Continuing Education.

Interessenkonflikt B. Schigl gibt an, dass kein Interessenkonflikt besteht.

Open Access Dieser Artikel wird unter der Creative Commons Namensnennung 4.0 International Lizenz (http:// creativecommons.org/licenses/by/4.0/deed.de) veröffentlicht, welche die Nutzung, Vervielfältigung, Bearbeitung, Verbreitung und Wiedergabe in jeglichem Medium und Format erlaubt, sofern Sie den/die ursprünglichen Autor(en) und die Quelle ordnungsgemäß nennen, einen Link zur Creative Commons Lizenz beifügen und angeben, ob Änderungen vorgenommen wurden.

\section{Literatur}

Abdul-Hussain, S. (2012). Genderkompetenz in Supervision und Coaching. Wiesbaden:VS Verlag.

Buchinger, P. (2015). Braucht Life Science Beratung? Empirische Untersuchung zu Bedarfund Inanspruchnahme von Beratung in Life Science Organisationen. Masterthesis 
Donau Universität Krems. http://webthesis.donau-uni. ac.at/thesen/94401.pdf.Zugegriffen: 13. März2013

Deutsche Gesellschaft für Supervision DGSv (2008). Der Nutzen von Supervision. http://d-nb.info/1006968954/ 34.Zugegriffen: 12. März2016

Ehrhardt, J. (2010). Verletzungen in der Supervision und in der Lehrsupervision. Diplomarbeit im Ergänzungsstudiengang „Diplom-Supervisor/in“. Amsterdam, Krems: Faculty of Human Movement Sciences Vrije Universiteit Amsterdam \& Donau Universität.

Ehrhardt, J., \& Petzold, H. G. (2011). Wenn Supervisionen schaden. Explorative Untersuchungen im Dunkelfeld „riskanter supervisorischer Praxis“. Integrative Therapie, 37(1-2), 137-192.

Gottfried, K. (2012). Supervision in Psychiatrischen Kliniken. Hamburg: Dr. Kovac.

Hausinger, B. (2009). Ausblicke. In R. Haubl, \& B. Hausinger (Hrsg.), Supervisionsforschung: Einblicke und Ausblicke (S. 241-249). Göttingen:Vandenhoeck \& Ruprecht.

Hinterwallner, H. (2010). (Un)Erwünschte Wirkungen von Supervision in der sozialen Arbeit. Diplomarbeit um Masterlehrgang sozialraumorientierte und klinische soziale Arbeit.Wien:FHCampus.

Jungmeier, B. (2011). Evangelische Pfarrer/innen und Supervision: Möglichkeiten und Grenzen des Beratungsformates Supervision zur Unterstützung und zur Burnout Prophylaxe von österreichischen Pfarrern und Pfarrerinnen eine Bestandsaufnahme und empirische Untersuchung. Masterthesis Donau Universität Krems. http://webthesis. donau-uni.ac.at/thesen/90445.pdf. Zugegriffen: 19. März2016

Karlinger, S. (2010). Gender matters. Zur Bedeutung der Genderkompetenz von Supervisorinnen und Supervisoren im psychosozialen Feld. Masterthesis Donau Universität Krems.http://webthesis.donau-uni.ac.at/thesen/90310. pdf.Zugegriffen: 12. März2016

Kero, J. (2010). Thematisierung von unerwünschten Effekten in der Supervision untersucht anhand der Quellenanalyse der Zeitschriften OSC und Supervision. www.fpi-publikation.de/images/stories/downloads/ supervision/kero_judith_monika-thematisierung supervision-07_2010.pdf.Zugegriffen:12.März2016

Keiblinger, M. (2012). Risiken, Schäden, Nebenwirkungen von Supervision aus Sicht von ExpertInnen. Masterthesis Donau Universität Krems. http://webthesis.donau-uni. ac.at/thesen/91322.pdf.Zugegriffen: 12. März2016

Kraus, D. R., Castonguay, L., Boswell, J. F., Nordberg, S., \& Hayes, J. (2011). Therapist effectiveness: Implications for accountability and patient care. Psychotherapy research, 21(3), 267-276.

Leitner, A., Petzold, H.G., Orth, S., Sieper, J., \& Telsemeyer, P. (2004). Mythos Supervision? Zur Notwendigkeit von „konzeptkritischen“ Untersuchungen im Hell- und Dunkelfeld zu Wirkungen, Nebenwirkungen, Risiken und Rechtsverletzungen in der supervisorischen Praxis. http://www.donau-uni.ac.at/imperia/md/content/ studium/umwelt_medizin/psymed/artikel/mythsu04. pdf.Zugegriffen: 12. März2016
Leitner, A., Schigl, B., \& Märtens, M. (2014). Wirkung, Risiken und Nebenwirkungen von Psychotherapie. ein Beipackzettel für TherapeutInnen und PatientInnen. Wien: facultas wuv.

Möller, H. (2012). Was ist gute Supervision? Grundlagen, Merkmale, Methoden. Kassel: University Press.

Petzold, H., Schigl, B., Fischer, M., \& Höfner, C. (2003). Supervision auf dem Prüfstand. Wirksamkeit, Forschung, Anwendungsfelder, Innovation. Opladen: Leske \& Budrich.

Petzold, H. G., Müller, L., \& König, M. (2008). Supervision im Feld der Altenarbeit im deutschsprachigen Raum - ein Vergleich der empirischen Felderkundungen in Österreich, Deutschland und der Schweiz. www.fpi-publikation.de/images/stories/downloads/ supervision/petzoldmuellerkoenigsup_09_2008.pdf.Zugegriffen: 13. März2016

Petzold, H. G. (2009). „Macht“, „Supervisorenmacht“ und „potentialorientiertes Engagement“. Überlegungen zu vermiedenen Themen im Feld der Supervision und Therapie verbunden mit einem Plädoyer für eine Kultur „transversaler und säkular-melioristischer Verantwortung“. http://www.fpi-publikation.de/images/stories/ downloads/supervision/petzold_macht_supervision_ 04_2009druck.pdf.Zugegriffen:19. März2016

Pühl, H. (Hrsg.). (2009). Handbuch der Supervision 3. Berlin: Leutner.

Schigl, B. (2004). Qualitätskriterien supervisorischen Handelns: Ausgewählte Ergebnisse einer Evaluation der internationalen Forschungsliteratur. In W. Fröhlich, \& W. Jütte (Hrsg.), Qualitätsentwicklung in der postgradualen Weiterbildung. Internationale Entwicklungen und Perspektiven (S.440-449). Münster:Waxmann.

Schigl, B. (2007). Wirkfaktoren und Qualitätskriterien von erfolgreichen Supervisionsprozessen: Eine qualitative Erhebung bei Expert/innen und Supervisand/innen. supervision, 2007(1), 41-49.

Schigl, B. (2011). Risiken, Nebenwirkungen und Schäden durch Supervision und Beratung. Integrative Therapie, 37(1-2), 113-137.

Schigl, B., \& Gahleitner, S. B. (2013). Fehler machen - aus Fehlern lernen? - Perspektiven zur Klassifizierung von psychotherapeutischen Fehlern und dem Umgang damit In: Psychotherapiewissenschaft. Jg. 3/1. http://www. psychotherapie-wissenschaft.info/index.php/psy-wis/ article/view/1001.Zugegriffen: 13. März2016

Schigl, B. (2014a). Wie gehen PsychotherapeutInnen mit ihren Fehlern um? In A. Leitner, B. Schigl, \& M. Märtens (Hrsg.), Wirkung Risiken und Nebenwirkungen von Psychotherapie(S.77-80). Wien: facultas-wuv.

Schigl, B. (2014b). We are all part of the game! Gender + Diversity-Kompetenz für psychosoziale Berufe. In S. B. Gahleitner, R. Reichel, B. Schigl, \&A. Leitner (Hrsg.), Wann sind wir gut genug? Selbstreflexion, Selbsterfahrung und Selbstsorge in Psychotherapie, Beratung und Supervision (S.62-73). Weinheim: Beltz-Juventa. 